\title{
Refractometric Sensing of Heavy Oils in Fluorescent Core Microcapillaries
}

\author{
V. Zamora, Z. Zhang and A. Meldrum* \\ Department of Physics, University of Alberta, Edmonton, AB, T6G2E 1 - Canada \\ e-mail: ameldrum@ualberta.ca \\ * Corresponding author
}

\begin{abstract}
Résumé - La détection réfractométrique des huiles lourdes dans les microcapillaires à cœur fluorescents - La détection par réfractométrie d'huiles lourdes calibrées (densité $>1000 \mathrm{~kg} / \mathrm{m}^{3}$ ) a été démontrée à l'aide de micro-capillaires à cœurs fluorescents. La surface interne d'un capillaire de 25 microns a d'abord été revêtue d'une couche de silicium fluorescent à haut indice de réfraction (fluorescent silicon Quantum Dots, QD). Ce film de QD permet le développement de résonances cylindriques en mode galerie (Whispering Gallery Mode, WGM) à l'intérieur du capillaire. Les huiles lourdes recouvrant une gamme étendue de l'indice de réfraction ont été injectées dans le canal du capillaire, provoquant de grands changements pour les longueurs d'onde de résonance WGM. La sensibilité des huiles lourdes aux indices de réfraction les plus élevés, est d'environ $250 \mathrm{~nm}$ par Unité d'Indice de Réfraction (nm/UIR), ce qui est la sensibilité la plus élevée observée jusqu'à présent pour un capteur réfractométrique fonctionnant en mode fluorescent. Cela est une indication que les microcapillaires à cœurs fluorescents pourraient être une alternative viable par microfluidique pour la détection chimique ou par réfractométrie lors des divers stades de la transformation, du contrôle et de l'utilisation du pétrole et du gaz.
\end{abstract}

\begin{abstract}
Refractometric Sensing of Heavy Oils in Fluorescent Core Microcapillaries - The refractometric sensing of calibrated heavy oils (density $>1000 \mathrm{~kg} / \mathrm{m}^{3}$ ) is demonstrated using fluorescent-core microcapillaries. A 25-micron capillary channel was first coated with a high-index layer of fluorescent silicon Quantum Dots (QD). This QD film supports the development of cylindrical Whispering Gallery Mode (WGM) resonances inside the capillary. Heavy oils spanning a wide range of refractive index were pumped into the capillary channel, causing large shifts in the fluorescence WGM resonant wavelengths. The sensitivity for heavy oils approached $250 \mathrm{~nm}$ per Refractive Index Unit (nm/RIU) at the higher oil indices, which is the highest sensitivity so far observed for a refractometric sensor operating in the fluorescence mode. This suggests that fluorescent core microcapillaries may be a viable microfluidic alternative for refractometric or chemical sensing in various stages of oil and gas processing, monitoring and usage.
\end{abstract}




\section{INTRODUCTION}

Microfluidic lab-on-a-chip optical analysis methods have advanced rapidly in recent years, to the point where commercial biochip analysis devices have reached the market [1]. These devices are attractive because they can provide measurements at the point-of-need, require microscopic amounts of analyte (e.g., $\mathrm{nL}$ volumes) and can be highly sensitive to trace quantities of analyte. Much of the interest in microfluidic sensors has so far been focused in the area of biochemistry. Optical devices have been created that can detect a variety of biomolecules ranging from single viruses [2] to DNA [3], pesticides [4], and other water-based solutes including sucrose [5, 6] and glucose [7]. However, progress in microfluidics relevant to the oil-and-gas industry has been much slower. Some of the relatively few recent examples include the microfluidic measurement of $\mathrm{CO}_{2}$ diffusivity in bitumen $[8,9]$, microfluidic oil-water phase separation [10], separation of polychlorinated biphenyls in transformer oil [11], microscale manipulation and transport of chemicals for subsequent geochemical analysis [12] and nanofiltration of crude oils [13]. At the current time, the capacity of microfluidics for sensing applications in the oil and gas field remains relatively little explored.

Thin-walled microcapillaries are an attractive option for microfluidic sensing applications. In these devices, referred to as Liquid-Core Optical Ring Resonators (LCORR) [14], the analyte fluid is pumped through the capillary channel while light from a tuneable laser is coupled evanescently through a fiber taper into the capillary wall. The wall of the capillary acts as a cylindrical cavity inside which high-quality-factor (high- $Q$ ) optical resonances can develop. These optical resonances are called Whispering Gallery Modes (WGM), named by Lord Rayleigh in 1912 after their acoustic analogue. The evanescent tail of the WGM interacts with the fluid medium (Fig. 1), leading to observable shifts in the resonance spectral position as a function of the refractive index of the analyte. However, LCORR are laborious to fabricate on a one-by-one basis, and they are fragile. Additionally, the requirement for a tuneable laser and nanopositioning equipment adds experimental cost to the system.

We recently developed a method that avoids these issues [6]. In this method, a layer of fluorescent silicon Quantum Dots (QD) is coated on the interior surface of the capillary, forming a film about $0.5 \mu \mathrm{m}$ thick. The high refractive index of the QD film $\left(n_{2}=\sim 1.67\right)$ supports total internal reflection at the boundary between the film and the capillary wall $\left(n_{3}=1.452\right)$, thus permitting the development of WGM concentrated in the film region. This method requires no thinning treatments and leads
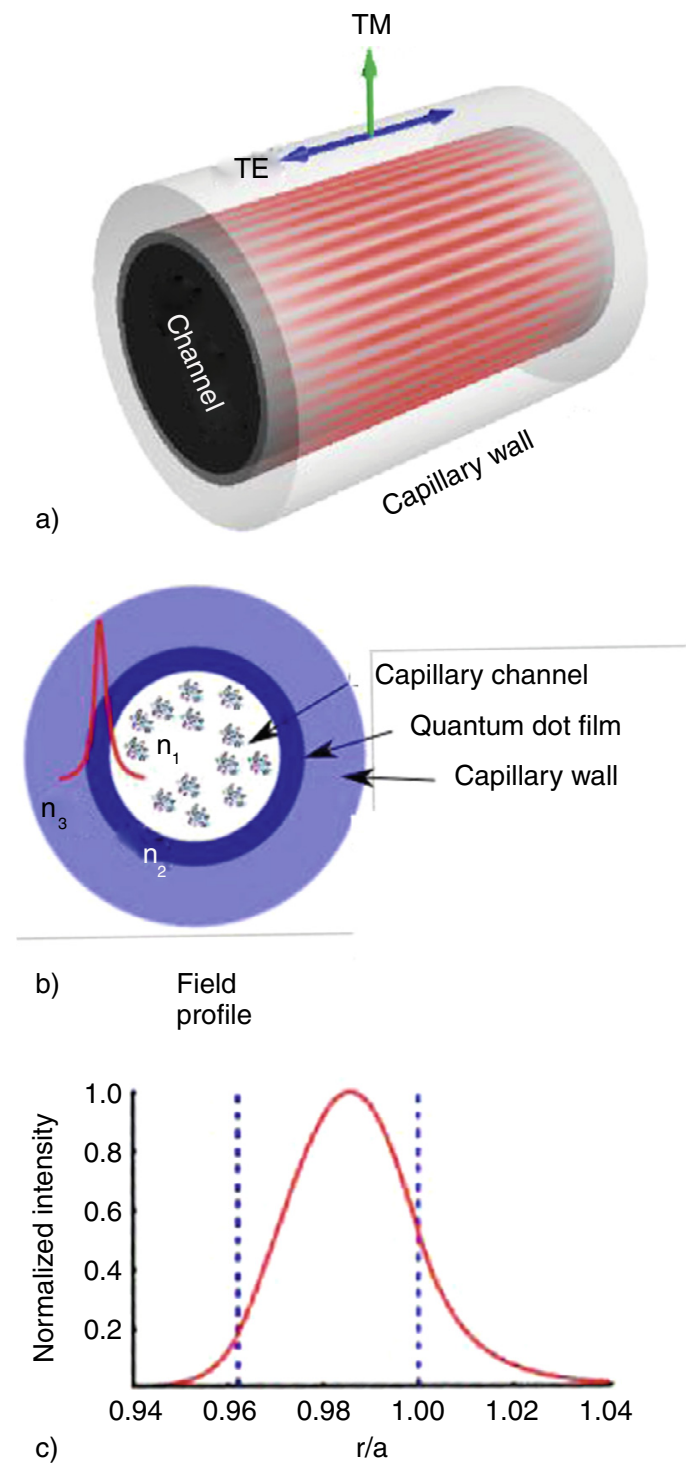

Figure 1

a) Drawing of the device structure, illustrating the development of the WGM in the film layer between the channel and the capillary wall. b) A cross sectional view illustrating the field intensity (red "wave") primarily confined within the QD layer of index $n_{2}$ but with a tail extending into the analyte (index $n_{1}$ ). (c) The electric field intensity distribution obtained by solving the three-layer system of cylindrical Bessel functions. The part of the E-field extending into the channel is responsible for the sensing action.

to a robust, durable, and reusable optical sensor that works via fluorescence instead of evanescent coupling through a fiber taper, as in LCORR (Fig. 1). Finally, instead of a tuneable laser, one simply needs to collect the fluorescence signal with a simple spectrometer.

The goal of the present work was twofold. First, we wanted to demonstrate whether these fluorescent core microcapillaries would work with heavy oils. Oils are 
considerably more viscous and are more difficult to handle than the typical water-based solutions. Second, we aimed to track the behavior of the optical modes when the refractive index of the oil in the channel approached or even exceeded the refractive index of the QD layer. This is generally not the case in biochemical sensors, where the channel refractive index, $n_{1}$, is usually close to 1.33 . The refractive index of crude oil varies considerably with composition but typical values are in the range of $n_{1}=1.45-1.50[15,16]$. Here, the refractive index will be varied from 1.33 to 1.75 , starting from water and moving to a set of calibrated heavy "Cargill oils" [17] that span a wide range in refractive index.

\section{EXPERIMENTAL METHOD}

The fluorescent core microcavities were fabricated using the methods we developed in [18]. Briefly, a solution of hydrogen silsesquioxane $\left(\mathrm{Si}_{8} \mathrm{H}_{8} \mathrm{O}_{12}\right)$ dissolved in methyl isobutyl ketone was drawn into a commercial silica capillary (PolyMicro Technologies) with a nominally 25-micron inner diameter. This is done by dipping the capillary into the solution and waiting a few seconds for the solution to flow into the channel by capillary forces. Many samples can be fabricated at the same time; however, only a single device was used for the work that follows. The capillary was then thermally processed at $1100^{\circ} \mathrm{C}$ in a nitrogen-hydrogen gas mixture, in order to evaporate the solvent and segregate the remaining film into a mixture of silicon QD embedded in an oxide matrix [6].

Next, the capillary, now with a layer of quantum dots coating the channel surface (see Fig. 1 for a diagram of the structure), was interfaced to a multi-channel syringe micropump that was loaded with a set of several different refractive index oils. Initially, water $\left(n_{1}=1.33\right)$ was pumped into the capillary channel and the QD were excited with an Ar-laser at an incident power of about $45 \mathrm{~mW}$. The resulting fluorescence was collected through a $10 \times$ objective lens with a numerical aperture of 0.22 and sent to a standard Czerny-Turner spectrograph for analysis. The spectra were calibrated for wavelength by using a $\mathrm{HgAr}$ lamp and for intensity by using a blackbody radiator (the LS1 light source from Ocean Optics). This process was repeated for all ten refractive index oils. Between each oil analysis, research grade methanol was pumped through the capillary for about 5 minutes, in order to clean the channel and remove oil residues from the previous measurement.

\section{RESULTS}

When the QD film was excited with the 488-nm laser line, a red fluorescence was readily visible, even to the naked eye.

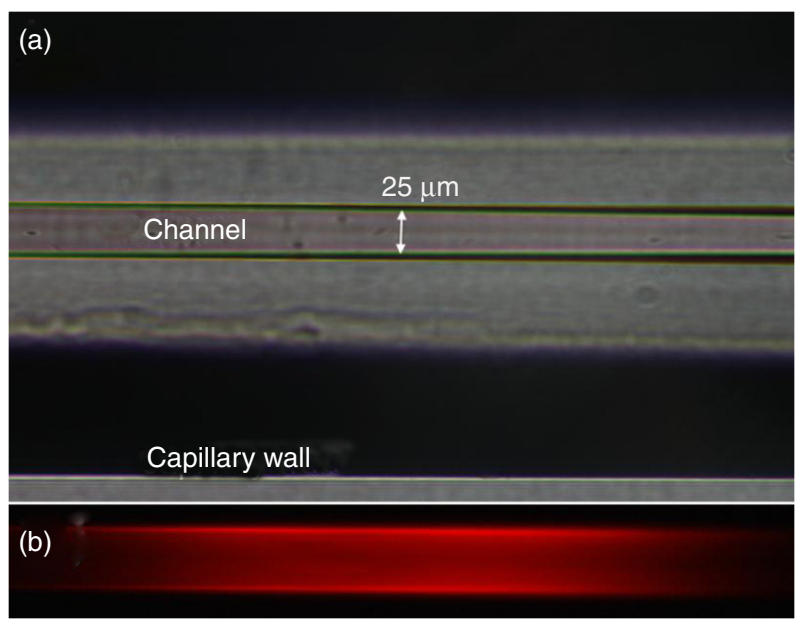

Figure 2

(a) Transmitted light image of a capillary whose channel surface is coated with a layer of quantum dots. The lower wall of the capillary is visible at the bottom of the image. (b) is a fluorescence image of the same capillary, showing the red-glowing QD in the channel region.

Figure 2 shows a microscope image of the whole capillary and a fluorescence image of the capillary channel. The bright red bands at the channel edges are consistent with the presence of the WGM. Welldeveloped WGM were indeed clearly visible in the fluorescence spectrum (Fig. 3). The resonances are characterized by:

- a higher visibility in the Transverse Electric (TE) polarization (i.e., with the electric field parallel to the capillary axis). This polarization phenomenon is sometimes reported in cylindrical resonators but is not fully understood [19];

- fluorescence $Q$ factors $\left(Q=\lambda_{\text {peak }} / \Delta \lambda_{\text {peak }}\right)$ of $\sim 500$;

- a Free Spectral Range (FSR) between fundamental resonances of $\sim 4.5 \mathrm{~nm}$, which is related mainly to the radius of the channel;

- a slight skewing of the resonances toward shorter wavelengths. This skewing effect is consistent with the propagation of spiralling modes having a component of the wavevector parallel to the capillary axis $[6,20]$.

The properties of the resonances depend on the refractive indices of the channel medium $\left(n_{1}\right)$, the film $\left(n_{2}\right)$, and the capillary wall $\left(n_{3}\right)$. For these experiments, the index of the channel fluid (standard oils) and silica capillary $\left(n_{3}=1.452\right)$ were considered known. The film refractive index can be estimated independently using spectroscopic ellipsometry methods on a flat QD film of known thickness. This yielded a refractive index of $n_{2}=1.67 \mathrm{in}$ the wavelength range of the WGM in Figure 3 [6]. 


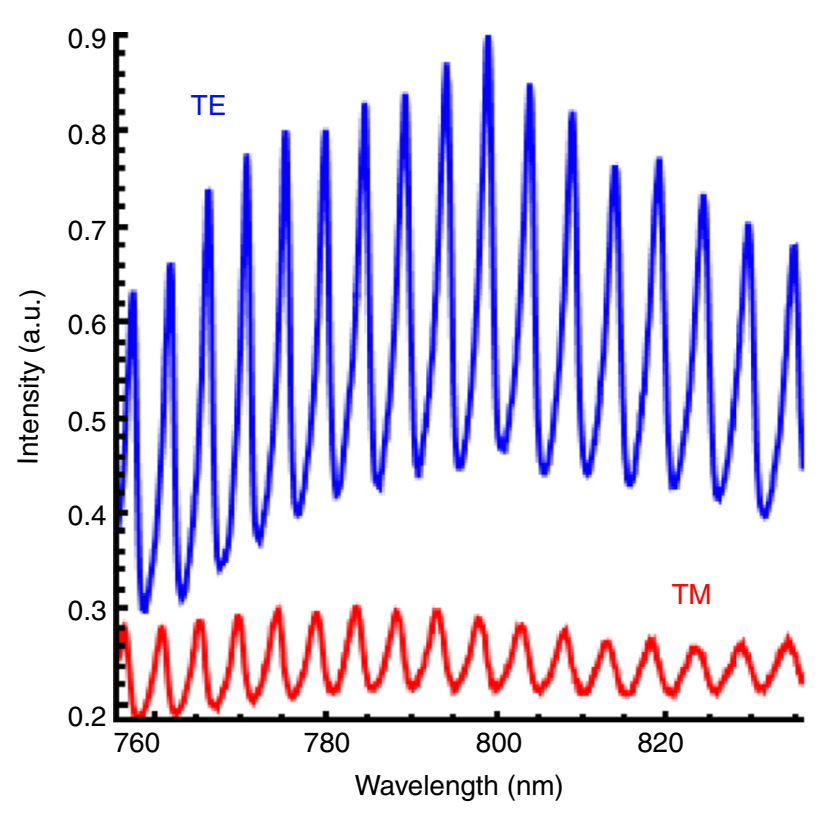

Figure 3

Fluorescence whispering gallery modes observed in the fluorescent capillary (i.e., the spectrum of the luminescence in Fig. 2b). The sharp peaks are the Transverse Electric (blue) and transverse magnetic (red) polarized WGM, offset for clarity.

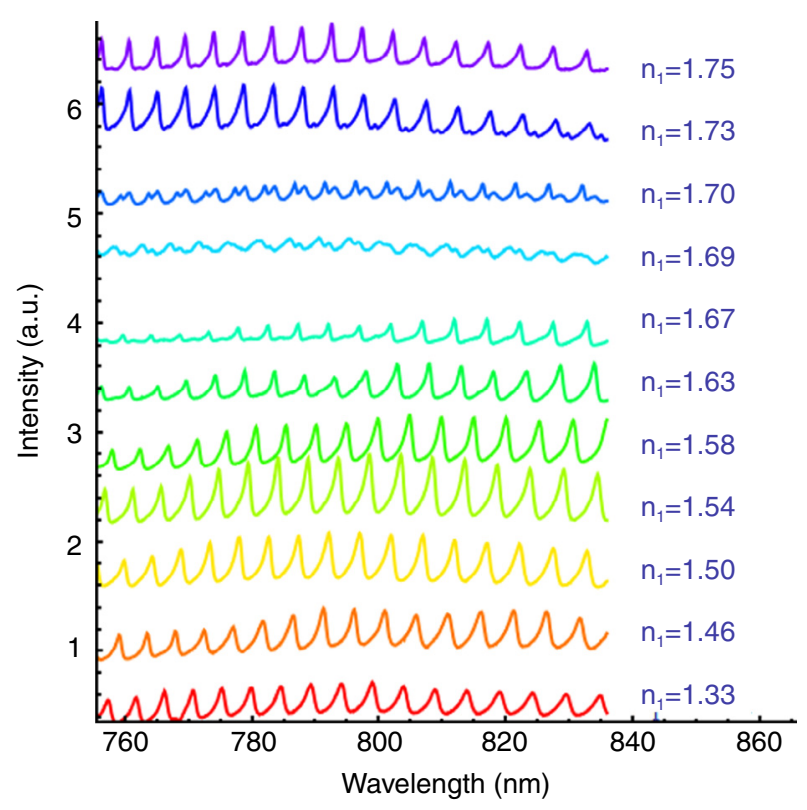

Figure 4

Evolution of the QD luminescence spectrum starting from water $\left(n_{1}=1.33\right)$ and subsequently pumping in oils with refractive indices ranging from 1.46 to 1.75 . Each spectrum is labeled with the nominal oil refractive index value for $\lambda=589.3 \mathrm{~nm}$.
Some variation is possible in a curved film inside a capillary, owing to minor differences in the evolution of the HSQ film during annealing inside a capillary [21].

The evolution of the WGM fluorescence spectrum with different refractive index oils in the capillary channel is shown in Figure 4. The resonances shift monotonically to longer wavelengths with increasing oil index $n_{1}$. As $n_{1}$ increases close to the value of the QD film, the mode spectrum evolves toward a complicated structure consisting of overlapping groups of spectral peaks. These are not combinations of different polarizations, since only the TE modes were collected by using a polarizer in the beam path. However, at the highest $n_{1}$ values, the fluorescence spectrum regains a more simple form consisting of a single strong family of maxima.

\section{MODELING}

In order to identify the resonances and determine the sensitivity of the device, we calculated both the predicted sensitivity and resonance wavevector from equations that describe the electric field in a layered cylinder [22]. Accordingly, the radial dependence of the field amplitude $E_{n, l}(r)$ for the $n^{\text {th }}$ radial order and the $l^{\text {th }}$ angular order is modeled in the standard form:

$$
E_{l}(r)=\left\{\begin{array}{l}
A_{l} J_{l}\left(n_{1} k r\right) \quad r<a-t \\
B_{l} J_{l}\left(n_{2} k r\right)+C_{l} Y_{l}\left(n_{2} k r\right) \quad a-t<r<a \\
D_{l} Y_{l}\left(n_{3} k r\right) \quad r>a
\end{array}\right.
$$

In Equation (1), $n_{1}, n_{2}$ and $n_{3}$ are the refractive indices of the channel, QD film and capillary wall, respectively, and $J_{l}$ and $Y_{l}$ are the $l^{\text {th }}$ cylindrical Bessel functions of the first and second kind. The boundary conditions for TE polarization (the field amplitude and its derivatives must be continuous) lead to the solutions for the resonance wavevectors $k$. These are given by the roots of the following expression [22]:

$$
\frac{n_{3} Y_{l}^{\prime}\left(n_{3} k a\right)}{n_{2} Y_{l}\left(n_{3} k a\right)}=\frac{\left(B_{l} / C_{l}\right) J_{l}^{\prime}\left(n_{2} k a\right)+Y_{l}^{\prime}\left(n_{2} k a\right)}{\left(B_{l} / C_{l}\right) J_{l}\left(n_{2} k a\right)+Y_{l}\left(n_{2} k a\right)}
$$

Here, the ratio of the prefactors is:

$$
\left(B_{l} / C_{l}\right)=\frac{n_{2} J_{l}\left(n_{1} k(a-t)\right) Y_{l}^{\prime}\left(n_{2} k(a-t)\right)-n_{1} J_{l}^{\prime}\left(n_{1} k(a-t)\right) Y_{l}\left(n_{2} k(a-t)\right)}{-n_{2} J_{l}\left(n_{1} k(a-t)\right) J_{l}^{\prime}\left(n_{2} k(a-t)\right)+n_{1} J_{l}^{\prime}\left(n_{1} k(a-t)\right) J_{l}\left(n_{2} k(a-t)\right)}
$$


In Equations $(2,3)$, the prime $\left({ }^{\prime}\right)$ indicates the derivative with respect to the argument. There are an infinite number of roots of Equation (2), corresponding to the different radial orders (i.e., the number of field intensity maxima in the radial direction, from the capillary center to the wall). Finally, the refractometric sensitivity, $S$ is [22]:

$$
S=\frac{\lambda_{0} I_{1} n_{1}}{I_{1} n_{1}^{2}+I_{2} n_{2}^{2}+I_{3} n_{3}^{2}}
$$

In Equation (4), $I_{1}, I_{2}$ and $I_{3}$ are related to the fractions of the field intensity in the three regions, and $\lambda_{0}$ is the resonance wavelength. Analytical formulae for the field profiles $I_{1}, I_{2}$ and $I_{3}$ can be calculated from the solutions of Equations (2-4). The resulting expressions are too lengthy to repeat here but are derived in full detail in $[22,23]$ for the nearly equivalent case of sphere.

Three examples of the electric field resonances and sensitivities calculated with these methods are shown in Figure 5. For a fluid refractive index of 1.33, only $2.8 \%$ of the first-order radial mode profile extends into the capillary channel, and its calculated sensitivity is $S=11 \mathrm{~nm} /$ RIU. As the fluid index increases, the electric field intensity is gradually "pulled" into the channel until, when the oil index is 1.75 , just over $62 \%$ of the field is in the analyte. For this fundamental mode, the calculated sensitivity reaches $309 \mathrm{~nm} / \mathrm{RIU}$. For a second-order radial mode (Fig. 5c), the sensitivity is $265 \mathrm{~nm} / \mathrm{RIU}$ for $n_{2}=1.75$ (i.e., slightly lower than for the first order mode).

The predicted theoretical sensitivities (Eq. 4) agree well with the experimental results: for example, experimentally the average sensitivity, defined as $\delta \lambda / \delta n$, is $22.5 \mathrm{~nm} / \mathrm{RIU}$ in the index range from 1.46 to 1.50 . At the mean index of 1.48 , Equation (4) gives $S=22.9 \mathrm{~nm} / \mathrm{RIU}$ for the above-listed parameters. In the high refractive index region, the experimental average sensitivity between $n_{1}=1.73$ and 1.75 is $240 \mathrm{~nm} / \mathrm{RIU}$; whereas, the theoretical value for the second order modes is $270 \mathrm{~nm} / \mathrm{RIU}$ at $n_{1}=1.74$. To our knowledge, $240 \mathrm{~nm} / \mathrm{RIU}$ is the highest refractometric sensitivity observed for fluorescent cavities, comparable in magnitude to some of the highest values in evanescently coupled devices [24, 25].

\section{SIMULATION}

In order to understand the evolution of the mode spectrum, 2D Finite-Different Time-Domain (FDTD)
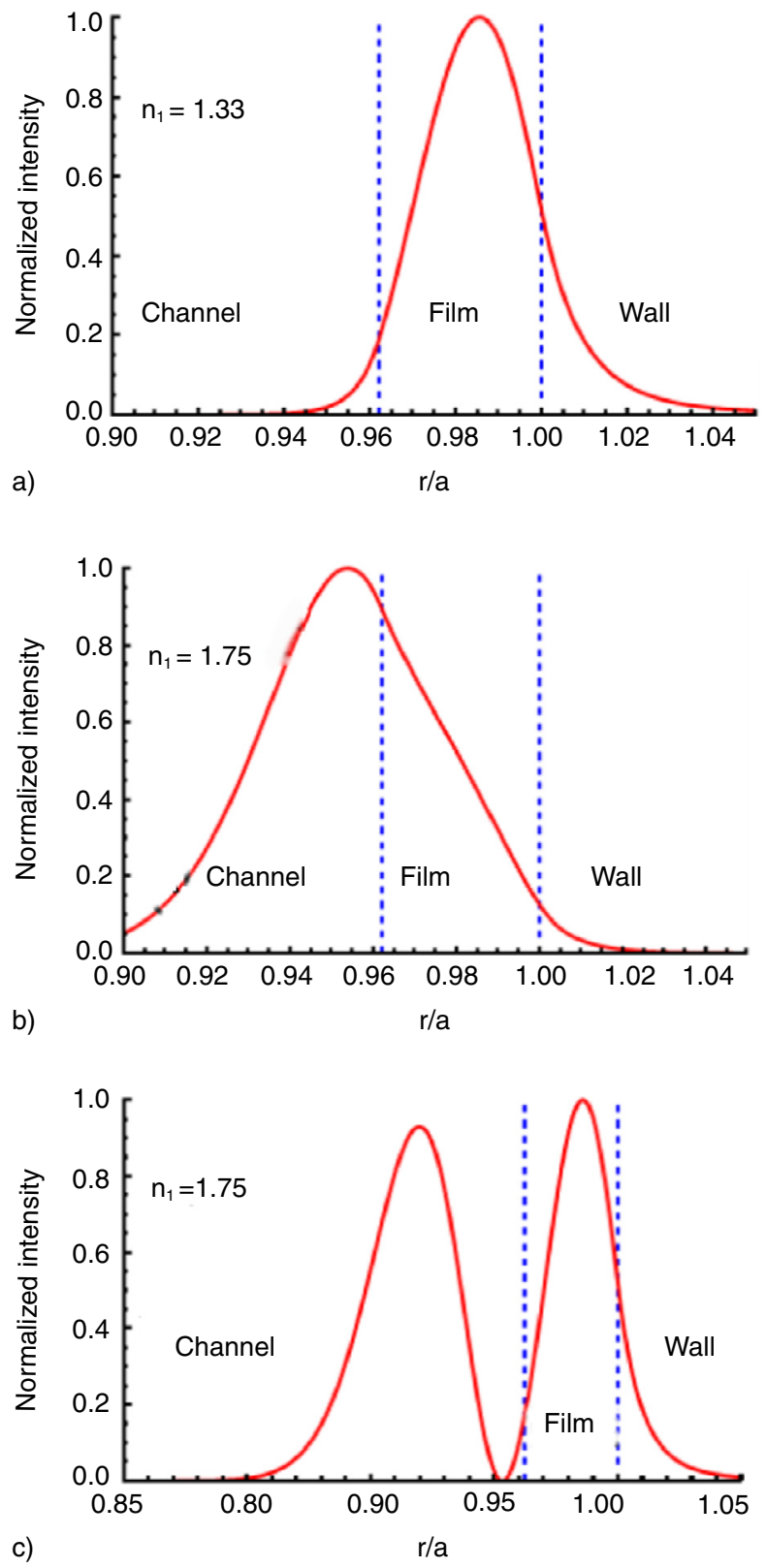

Figure 5

Electric field intensity profile for the $n=1, l=155$ mode when a) the channel fluid is water $\left(n_{1}=1.33\right)$; and b) when the channel fluid is refractive index oil $\left(n_{1}=1.75\right)$. c) shows the $n=2, l=155$ mode for a channel index of 1.75. Upon going from (a) to (b), the field maximum is drawn into the channel region, increasing the theoretical sensitivity from $11.2 \mathrm{~nm} / \mathrm{RIU}$ to $308.4 \mathrm{~nm} / \mathrm{RIU}$. The calculated sensitivity in c) is $265 \mathrm{~nm} / \mathrm{RIU}$. In all cases, the capillary inner diameter was $25 \mu \mathrm{m}$, and the refractive index and thickness of the film were 1.675 and $0.475 \mu \mathrm{m}$, respectively.

simulations were performed using starting positions obtained from the solutions of Equation (2). Essentially, the film index and thickness and the capillary diameter 
were allowed to vary slightly until the results closely modeled the observed spectra. Then, these parameters were used in the full FDTD simulations in order to model the complete wavelength spectrum. The nominal oil refractive indices are given by the manufacturer for a calibration wavelength of $589 \mathrm{~nm}$. The FDTD simulations accounted for index dispersion by using the known Cauchy constants for each oil composition. The established procedures were followed for the simulations, with respect to grid size, matching layers, and number of time steps [26].

A good agreement between the theory (Eq. 1-3) and experiment was found for a film thickness of $475 \mathrm{~nm}$, a film Refractive Index (RI) of 1.675, and a capillary inner radius of $12.5 \mu \mathrm{m}$. This RI agrees closely with that found by ellipsometry. Employing these values in the FDTD simulations provided in general a good match with experiment (Fig. 6). Three representative spectra are compared against their experimental counterparts, spanning the range of index where $n_{1}<n_{2}$ (Fig. 6a), $n_{1} \approx n_{2}$ (Fig. 6b), and $n_{1}>n_{2}$ (Fig. 6c). Furthermore, the mode numbers can be identified in FDTD by performing a continuous wave simulation at the resonance wavelength.

The FDTD simulations show the reason for the evolution of the modes observed experimentally. At the lower oil refractive indices, the spectrum consists of a family of first-order radial modes. When the oil index approached the film index, a family of second-order radial modes appears and grows stronger with increasing $n_{1}$. At the same time, the first order modes weakened and grew less visible. When $n_{1} \approx n_{2}$, both families of WGM are present in nearly equal intensity. By the highest oil index, the first order radial modes had essentially vanished, and the spectrum consisted only of the second order modes. Thus, by "pulling" the field toward the core, the first order modes were suppressed and the second order ones were strongly enhanced.

\section{DISCUSSION}

There are currently few competitive solutions in the market for real-time in situ testing and monitoring of oil and gas production, storage and end-usage [8]. Gas chromatography and mass spectroscopy are still among the most commonly used analysis methods in the industry; both require large and expensive laboratory equipment located far from the point-of-need [27]. Microfluidic analysis methods have not yet been widely investigated for these applications; however, as shown here, capillary-type microfluidic structures can be used to analyze the optical properties of heavy oils. For a sensitivity of
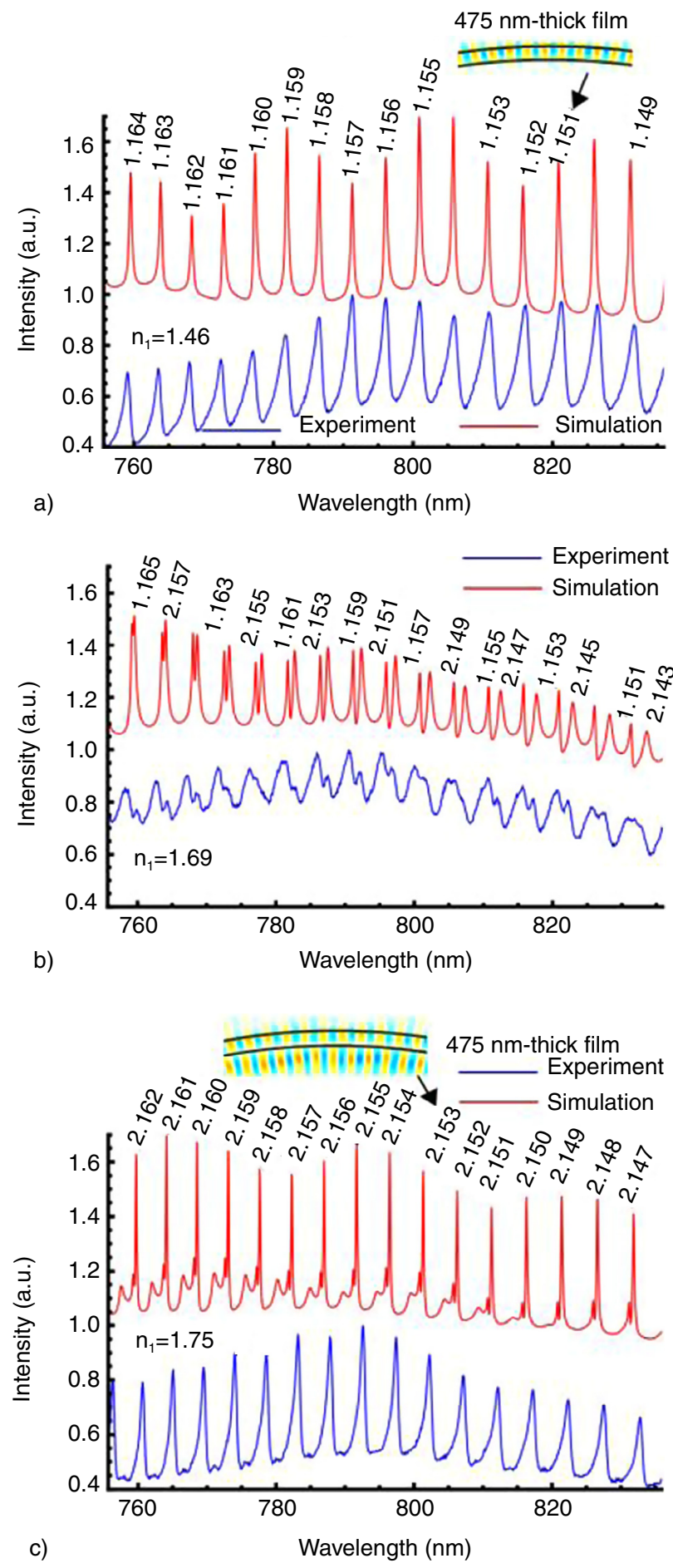

Figure 6

Comparison of FDTD simulations (red spectra) with experiment (blue spectra), labeled according to the nominal oil index $(1.46,1.69$ and 1.75$)$ in a) to label 1.46. For the simulations, the film thickness was $475 \mathrm{~nm}$ and its refractive index was 1.675. The mode numbers $n, l$ are labeled above each peak. The insets in a) and c) show part of the field distribution for the associated modes, with the dark lines indicting the film. The $Q$-factors are lower in the experiment mainly because of spiraling mode losses that cannot be simulated in 2 dimensions. 
$50 \mathrm{~nm} / \mathrm{RIU}$, the detection limits for heavy oils are on the order of $10^{-4} \mathrm{RIU}$, assuming $10 \mathrm{pm}$ spectral resolution is achievable via Fourier methods [28].

The results show that this sensor structure can be used for viscous heavy oils. Commercial hydrocarbon products feature many examples in which detection of impurities is important [29]. This work used optical calibration oils of known composition and refractive index in order to establish feasibility with heavy oils generally. Crude oils (as an example) are more complicated and have variable chemistry. Although crude oil is only one such example, we here highlight some potential advantages and issues with respect to the application of microfluidic sensors of the type developed in this work.

In order to know whether such sensors could handle the high viscosity of crude oils, the specifications of calibrated oils used in the present work are given. The kinematic viscosity of the oils varied from $\sim 2 \times 10^{-5}$ to $2 \times 10^{-6} \mathrm{~m}^{2} / \mathrm{s}$, the densities ranged from $832-3229 \mathrm{~kg} / \mathrm{m}^{3}$, and the viscosities varied from 0.006 to $0.015 \mathrm{~Pa} \cdot \mathrm{s}$. Thus, the viscosities tested are more typical of light crude oils and petroleum products than heavy crudes [30], which can have viscosities 100 times greater than these values. More viscous fluids than those tested could certainly be measured in the device, but the pumping time for fluids having the densities associated with heavy crudes would need further testing. As discussed below, the use of larger capillaries would alleviate viscosity issues to some degree.

In addition, crude oils may contain compounds that could "autofluoresce" within the capillary during measurement. Crude oil fluorescence originates primarily from the aromatic hydrocarbons [31] and is mainly controlled by the concentration of these compounds in the oil, as well as by the concentration of compounds that can quench the fluorescence. Light crudes tend to have stronger and narrower fluorescence spectra, as compared to heavy crudes [32, 33]. However, autofluorescence has no effect on the optical resonance structure of the WGM. In fact, hydrocarbon autofluorescence could potentially enhance performance by providing a stronger fluorescence signal intensity in the modes. Moreover, any compounds that quench the hydrocarbon fluorescence will not have any effect on the QD emission, since they are embedded within the silica capillary wall and are not exposed to the analyte.

The effect of insoluble particulate matter in oil products could be a limiting issue for this sensor, because they may block the capillary entrance. Asphaltene particles in crude oils from Alberta, Canada, for example, have been reported to range from $\sim 4$ to almost $300 \mu \mathrm{m}$ in mean diameter, depending on the heavy crude composition [34]. In this work, the inner capillary diameter was
$25 \mu \mathrm{m}$, although we have tested devices with inner diameters as large as $100 \mu \mathrm{m}$. The maximum diameter that can be used is limited in principle by the ability to resolve the WGM in the QD fluorescence spectrum. For larger capillaries, the WGM become more abundant and closely spaced, and they would eventually become unresolvable. In order to avoid severe aliasing, the mode spacing must be larger than at least twice the pitch of the spectrometer used. For a capillary diameter of $300 \mu \mathrm{m}$, Equations (1-3) give a mode spacing of $\sim 0.2 \mathrm{~nm}$, about twice the $0.1 \mathrm{~nm}$ pitch of our spectrometer. Thus, $300 \mu \mathrm{m}$ particulates push beyond the range of size possible for this type of sensor, unless a higher-resolution instrument is used to measure the fluorescence. Conversely, since the sensor requires only a tiny volume of analyte, prefiltration of a small quantity of product should be relatively simple. Using larger capillaries could alleviate viscosity issues as well.

An additional issue could be adsorption of crude oil components onto the capillary walls. This could be either harmful or beneficial to device performance. If, for example, solid particulates such as asphaltenes adsorb to the capillary wall, they would interfere with the refractometric measurements of the channel fluid. Conversely, adsorption of liquid components can be desirable, since this presents a method for analyte-specific detection. For example, the hydrophilic silica-based channel surfaces of these structures should adsorb water present either as an emulsion or dissolved in the hydrocarbon. Since the sensitivity of the device is greatest at the region near the channel walls, the device would be especially sensitive for such preferentially adsorbed compounds. In fact, the behavior of oil-water mixtures in the presence of silica surfaces has been widely investigated with respect to the effect of sand grains in such mixtures [35]. Treating the surface with polydimethylsiloxanes (for example) could also make the channel surface hydrophobic and thereby more sensitive to the reverse situation: detection of hydrocarbons in groundwater. Finally, fluorescence absorption due to components of the analyte could be problematic but could also provide an alternative sensing mechanism, since absorption or scattering losses will control the width of the resonances shown in Figure 6.

\section{CONCLUSION}

This work demonstrated the refractometric sensing of calibrated heavy oils pumped into microfluidic fluorescent-core capillary channels. The fluorescence spectra showed well-developed optical resonances that were highly sensitive to the refractive index of the oil in the channel. At the lower oil indices (i.e., $\left.n_{1}<1.6\right)$ the spectra 
were dominated by the first-order radial modes, having refractometric sensitivities on the order of several tens up to about $100 \mathrm{~nm}$ per refractive index unit. With increasing oil index, the first-order modes gradually disappeared while the second order ones became strong. The sensitivities in this region reached $240 \mathrm{~nm} / \mathrm{RIU}$, which is amongst the highest yet seen for a fluorescencebased optical resonance detection device. The results agreed well with theory and simulation and suggest that even higher sensitivity could be achieved by controlling the film thickness in these devices. The detection limits for crude oil would be conservatively in the range of $10^{-4}$ RIU, suggesting that such structures could be sensitive to low levels of unwanted impurities such as water or other trace compounds, or if the appropriate functionalization treatments can be developed.

\section{REFERENCES}

1 http://fluidicmems.com/list-of-microfluidics-lab-on-a-chipand-biomems-companies/, http://www.affymetrix.com/ estore/, http://www.micronit.com/ or http:// www.fluidigm. $\mathrm{com} /$

2 Vollmer F., Arnold S. (2008) Single Virus Detection from the Reactive Shift of a Whispering-Gallery Mode, PNAS 105, 20701-20704.

3 Suter J.D., White I.M., Zhua H., Shi H., Caldwell C.W., Fan X. (2008) Label-Free Quantitative DNA Detection Using the Liquid Core Optical Ring Resonator, Biosensors Bioelectronics 23, 1003-1009.

4 Yang G., White I.M., Fan X. (2008) An Opto-Fluidic Ring Resonator Biosensor for the Detection of Organophosphorus Pesticides, Sens. Actuators B: Chem. 133, 105-112.

5 Schweinsberg A., Hocdé S., Lepeshkin N.N., Boyd R.W., Chase C., Fajardo J.E. (2007) An Environmental Sensor Based on an Integrated Optical Whispering Gallery Mode Disk Resonator, Sens. Actuators B: Chem. 123, 727-732.

6 Manchee C.P.K., Zamora V., Silverstone J.W., Veinot J.G.C., Meldrum A. (2011) Refractometric Sensing with Fluorescent-Core Microcapillaries, Optics Express 19, 21540-21551.

7 Bianucci P., Rodriguez J.R., Clement C.M., Veinot J.G.C., Meldrum A. (2009) Silicon Nanocrystal Luminescence Coupled to Whispering Gallery Modes in Optical Fibers, J. Appl. Phys. 105, 023108.

8 Tavernier E., Sellier J., Marty F., Tabeling P., Bourouina T. (2008) Microsystem with fluidic and optical interface for inline measurement of $\mathrm{CO}_{2}$ in oil fields, IEEE Sens. $J$. 8, 430-434.

9 Fadaei H., Scarff B., Sinton D. (2011) Rapid MicrofluidicsBased Measurement of $\mathrm{CO}_{2}$ Diffusivity in Bitumen, Energy Fuels 25, 4829-4835.

10 Angelescu D.E., Mercier B., Siess D., Schroeder R. (2010) Microfluidic Capillary Separation and Real-Time Spectroscopic Analysis of Specific Components from Multiphase Mixtures, Anal. Chem. 82, 2412-2420.
11 Aota A., Date Y., Terakado S., Sugiyama H., Ohmura N. (2011) Analysis of Polychlorinated Biphenyls in Transformer Oil by Using Liquid-Liquid Partitioning in a Microfluidic Device, Anal. Chem. 83, 7834-7840.

12 Bowden S.A., Monaghan P.B., Wilson R., Parnell J., Cooper J.M. (2006) The Liquid-Liquid Diffusive Extraction of Hydrocarbons from a North Sea Oil Using a Microfluidic Format, Lab-on-a-Chip 6, 740-743.

13 Tsang M.-J., Ching M., Pomerantz A.E., Ballard Andrews A., Dryden P., Schroeder R., Mullins O.C., Harrison C. (2010) On the Nanofiltration of Asphaltene Solutions, Crude Oils, and Emulsions, Energy Fuels 24, 5028-5037.

14 White I.M., Oveys H., Fan X. (2006) Liquid-Core Optical Ring-Resonator Sensors, Opt. Lett. 9, 1319-1321.

15 Castillo J., Gutierrez H., Ranaudo M., Villarroel O. (2010) Measurement of the Refractive Index of Crude Oil and Asphaltene Solutions: Onset Flocculation Determination, Energy Fuels 24, 492-495.

16 El Ghandoora H., Hegazi E., Nasser I., Behery G.M. (2003) Measuring the Refractive Index of Crude Oil Using a Capillary Tube Interferometer, Opt. Laser Technol. 35, 361-367.

17 http://www.cargille.com/

18 Rodriguez J.R., Bianucci P., Meldrum A., Veinot J.G.C. (2008) Whispering Gallery Modes in Hollow Cylindrical Microcavities Containing Silicon Nanocrystals, Appl. Phys. Lett. 92, 131119.

19 Rakovich Y.P., Balakrishnan S., Donegan J.F., Perova T.S., Moore R.A., Gun'ko Y.K. (2007) The Fabrication, Fluorescence Dynamics, and Whispering Gallery Modes of Aluminosilicate Microtube Resonators, Adv. Funct. Mater. 17, 1106-1114.

20 Poon A.W., Chang R.K., Lock J.A. (1998) Spiral Morphology-Dependent Resonances in an Optical Fiber: Effects of Fiber Tilt and Focused Gaussian Beam Illumination, Opt. Lett. 23, 1105-1107.

21 Hessel C.M., Summers M.A., Meldrum A., Malac M., Veinot J.G.C. (2007) Direct Patterning, Conformal Coating, and Erbium Doping of Luminescent nc-Si/SiO $\mathrm{S}_{2}$ Thin Films from Solution Processable Hydrogen Silsesquioxane, Adv. Mater. 19, 3513-3516.

22 Teraoka I., Arnold S. (2006) Enhancing Sensitivity of a Whispering Gallery Mode Microsphere Sensor by a High-Refractive Index Surface Layer, J. Opt. Soc. Am. B: Opt. Phys. 23, 1434-1441.

23 Teraoka I., Arnold S., Vollmer F. (2003) Perturbation Approach to Resonance Shifts of Whispering-Gallery Modes in a Dielectric Microsphere as a Probe of a Surrounding Medium, J. Opt. Soc. Am. B 20, 1937-1946.

24 Zamora V., Díez A., Andrés M.V., Gimeno B. (2007) Refractometric Sensor Based on Whispering-Gallery Modes of Thin Capillaries, Optic. Express 15, 12011-12016.

25 Li H., Fan X. (2010) Characterization of Sensing Capability of Optofluidic Ring Resonator Biosensors, Appl. Phys. Lett. 97, 011105.

26 Boriskin A.V., Boriskina S.V., Rolland A., Sauleau R., Nosich A.I. (2008) Test of the FDTD Accuracy in the Analysis of the Scattering Resonances Associated With High-Q Whispering-Gallery Modes of a Circular Cylinder, J. Opt. Soc. Am. A, 25, 1169-1173. 
27 Lewis P.R., Maginell P., Adkins D.R., Kottenstette R.J., Wheeler D.R., Sokolowski S.S., Trudell D.E., Byrns J.E., Okandan M., Bauer J.M., Manley R.G., Frye-Mason C. (2006) Recent Advancements in the Gas-Phase Microchemlab, IEEE Sens. J. 6, 784-795.

28 Silverstone J.W., McFarlane S., Manchee C.P.K., Meldrum A. (2012) Ultimate Resolution for Refractometric Sensing With Whispering Gallery Mode Microcavities. Optics Express 20, 8284-8295.

29 Wiedemann L.S.M., d'Avila L.A., Azevedo D.A. (2005) Adulteration detection of Brazilian gasoline samples by statistical analysis, Fuel 84, 467-473.

30 Al-Besharah J.M., Salman O.A., Akashah S.A. (1987) Viscosity of Crude Oil Blends, Ind. Eng. Chem. Res. 26, 2445-2449.

31 Steffens J., Landulfo E., Coronato Courrol L., Guardani R. (2011) Application of Fluorescence to the Study of Crude Petroleum, J. Fluoresc. 21, 859-864.
32 Downare T.D., Mullins O.C. (1995) Visible and near-infrared fluorescence of crude oils, Appl. Spectrosc. 49, 754-764.

33 Ralston C.Y., Wu X., Mullins O.C. (1996) Quantum yields of crude oils, Appl. Spectrosc. 50, 1563-1568.

34 Ferworn K.A., Svrcek W.Y., Mehrotra A.K. (1993) Measurement of Asphaltene Particle Size Distributions in Crude Oils Diluted with $n$-Heptane, Ind. Eng. Chem. Res. 32, 955-959.

35 Langevin D., Poteau S., Hénaut I., Argillier J.F. (2004) Crude Oil Emulsion Properties and their Application to Heavy Oil Transportation, Oil Gas Sci. Technol. 59, 511-521.

Manuscript accepted in February 2013 Published online in September 2013 